\title{
OPEN Diagnostic and prognostic value of FDG PET-CT in patients with suspected recurrent thymic epithelial tumors
}

\author{
Guozhu Hou ${ }^{1,2}$, Yuanyuan Jiang ${ }^{1,2}$, Fang $\mathrm{Li}^{1,2} \otimes$ \&uying Cheng ${ }^{1,2 \bowtie}$
}

This study aimed to evaluate the diagnostic and prognostic value of FDG PET/CT in patients with suspected recurrent thymic epithelial tumors (TETs). We retrospectively reviewed 83 patients with histopathologically proven TETs (50 thymomas; 33 thymic carcinomas) who underwent FDG PET/CT after surgery. The sensitivity and specificity of FDG PET/CT in detecting recurrence were calculated. The progression-free survival rate (PFS) was calculated by the Kaplan-Meier method. FDG PET/CT results were positive in 50 patients and negative in 33. Recurrent TETs were confirmed in 40 of 50 patients with positive PET/CT findings. The sensitivity and specificity of FDG PET/CT were $100 \%$ and $76.7 \%$, respectively. Disease progression occurred in 28 patients during the follow-up. FDG PET/CT showed added prognostic value over the Masaoka stage and histopathology. Among patients with the same Masaoka stage, negative PET/CT was significantly associated with better PFS $(P<0.001)$. Similarly, among patients with the same histopathology, negative PET/CT was also associated with a significantly longer PFS $(P<0.001)$. FDG PET/CT demonstrated a good diagnostic performance in patients with recurrent TETs and had an important prognostic value in assessing the risk of disease progression.

Thymic epithelial tumors (TETs), including thymoma and thymic carcinoma, represent the most common primary neoplasms in the anterior mediastinum ${ }^{1}$. According to the World Health Organization (WHO) histological classification system, TETs are classified into thymomas and thymic carcinomas ${ }^{2}$. Thymomas are further divided into five different subtypes (A, AB, B1, B2, and B3). The prognosis of patients with thymomas is progressively worse from type $\mathrm{A}$ to $\mathrm{B}^{3}$. Compared with thymoma, thymic carcinoma shows even more aggressive clinical behavior with a worse prognosis ${ }^{4}$. Another important prognostic factor for TETs is the Masaoka staging system, which is the most adopted staging system in this entity and is based on the level of invasion ${ }^{5}$.

Surgery is the mainstay of treatment for TETs, and complete resection is one of the most important prognostic factors. TETs are known to have a possibility of recurrence even after complete resection. In the research of the Japanese Association for research on thymus, among 2835 patients who received surgical resection of TETs, $420(14.8 \%)$ developed recurrence ${ }^{6}$. In the study of the Japanese Association for chest surgery, $7.8 \%$ of patients with thymoma experienced recurrence ${ }^{7}$. In thymic carcinoma, $51 \%$ of patients developed recurrence ${ }^{7}$. Most recurrences are local and regional ${ }^{8,9}$, distant recurrence occurs in less than $5 \%$ of the cases ${ }^{10}$. Early detection of recurrence is necessary, because further treatment contributed to higher survival rates ${ }^{6}$.

FDG PET/CT has become an important tool for the diagnosis, staging, and re-staging of malignant tumors. FDG PET/CT is widely used in preoperative evaluation of TETs, and there is a good correlation between tumor metabolic activity and histopathological classification ${ }^{11-17}$. Its performance in detecting recurrent lesions and the ability to evaluate curative effects have also been reported in the literature ${ }^{18-20}$. Still, its impact on prognosis during the restaging process remains to be elucidated. Therefore, the aims of this study were: (1) to investigate the diagnostic performance of FDG PET/CT for suspected recurrent TETs, (2) to evaluate the added prognostic role of FDG PET/CT associated with other clinical parameters (histopathology and Masaoka stage) in the restaging process.

${ }^{1}$ Department of Nuclear Medicine, State Key Laboratory of Complex Severe and Rare Diseases, Peking Union Medical College Hospital, Chinese Academy of Medical Sciences and Peking Union Medical College, Beijing 100730, China. ${ }^{2}$ Beijing Key Laboratory of Molecular Targeted Diagnosis and Therapy in Nuclear Medicine, Beijing 100730, China.『email: lifang@pumch.cn; cwypumch@126.com 


\begin{tabular}{|c|c|}
\hline Characteristic & Value \\
\hline \multicolumn{2}{|l|}{ Age, years } \\
\hline Median & 52 \\
\hline Range & $19-83$ \\
\hline Gender, men/women, $\mathrm{n}$ & $41 / 42$ \\
\hline \multicolumn{2}{|l|}{ Follow-up duration, months } \\
\hline Median & 20 \\
\hline Range & $2-118$ \\
\hline \multicolumn{2}{|l|}{ Histopathology, n (\%) } \\
\hline Thymoma & $50(60.2)$ \\
\hline AB type & 9 \\
\hline B1 type & 10 \\
\hline B2 type & 14 \\
\hline B3 type & 11 \\
\hline Unclassified & 6 \\
\hline Thymic carcinoma & $33(39.8)$ \\
\hline \multicolumn{2}{|l|}{ Masaoka stage, $n(\%)^{\mathrm{a}}$} \\
\hline Stage I-II & $23(36.5)$ \\
\hline Stage III-IV & $40(63.5)$ \\
\hline \multicolumn{2}{|l|}{ Adjuvant treatment, $n$} \\
\hline Chemotherapy & 7 \\
\hline Radiotherapy & 27 \\
\hline Chemoradiation & 28 \\
\hline Disease progression, $\mathrm{n}(\%)$ & $28(33.7)$ \\
\hline \multicolumn{2}{|l|}{ PET/CT result, n (\%) } \\
\hline Negative & $33(39.8)$ \\
\hline Positive & $50(60.2)$ \\
\hline
\end{tabular}

Table 1. Characteristics of patients. ${ }^{a}$ Available in 63 patients.

\section{Results}

Patients. A total of 83 patients ( 41 men, 42 women) met the inclusion criteria and were included in this study. The clinical characteristics of patients were summarized in Table 1 . The median age was 52 years (range, 19-83; mean 52.2 \pm 12.9 ). All patients underwent surgery to remove the primary thymic tumor. Sixty-two patients also received adjuvant treatment in addition to surgery, including chemotherapy $(\mathrm{n}=7)$, radiotherapy $(n=27)$, chemoradiation $(n=28)$. The primary tumor histopathological types were thymoma in 50 patients and thymic carcinoma in 33 patients. Masaoka staging information at the time of diagnosis were available in 63 patients (stage I-II, $\mathrm{n}=23$; III-IV, $\mathrm{n}=40$ ).

FDG PET/CT performance. FDG PET/CT showed a positive finding in 50 patients and a negative finding in 33 patients. The findings of FDG PET/CT were histopathologically confirmed in 24 patients and were compared with clinical and/or imaging evaluation in the remaining 59. Forty patients with positive scans were confirmed with recurrent disease and were thus defined as truly positive. Ten patients were false positive, 33 patients were truly negative, and no patients in our series were false negative. The sensitivity, specificity, PPV, and NPV, and accuracy were $100 \%, 76.7 \%, 80 \%, 100 \%$, and $87.9 \%$, respectively. False-positive findings were related to post-surgical related uptake, and concomitant inflammatory disease affecting lymph nodes and lungs.

As described in Table 2, the most common mode of recurrence was regional recurrence, of which pleura was the most frequent site (Fig. 1). Of note was that the patients with distant sites of recurrence (liver, bone, lung) were all those with thymic carcinoma. Among the 40 patients with true positive FDG PET/CT findings, the SUVmax of the recurrent lesion with maximum uptake ranged from 2.4 to 27.5 (median, 7.0; mean, 7.95 \pm 4.77 ). The lesion SUVmax in patients with thymic carcinoma (mean, 10.20 5 .77; range, 3.4-27.5) was found to be significantly higher than that in patients with thymoma (mean $6.45 \pm 3.32$; range, $2.4-15.5 ; P=0.013$ ).

FDG PET/CT and outcome. After a median follow-up period of 20 months, 28 patients had disease progression. Among the remaining 55 patients without disease progression, 12 had stable disease and 43 were disease-free. The 2-year and 3-year PFS rate was summarized in Table 3. By adding PET/CT-derived information (positive vs. negative) to Msaoka atage (I/II vs. III/IV) and histopathology (thymic carcinoma vs. thymoma), there was allowed stratification of the risk of disease progression (Fig. 2). Among 23 patients with Masaoka stages I and II, 6 patients were PET/CT positive, and patients with negative PET/CT scans had longer PFS than those with positive scans $(\mathrm{P}=0.075$; Fig. $2 \mathrm{a})$. Among 40 patients with Masaoka stage III and IV, 24 patients were 


\begin{tabular}{|c|c|}
\hline Sites of recurrence & Frequency $(\mathbf{n})^{\mathrm{a}}$ \\
\hline Regional recurrence & 36 \\
\hline Pleura & 29 \\
\hline Pericardium & 6 \\
\hline Lymph node & 9 \\
\hline Local & 5 \\
\hline Liver & 6 \\
\hline Lung & 1 \\
\hline Bone & 2 \\
\hline
\end{tabular}

Table 2. Sites of recurrence on FDG PET/CT. a Some of the patients had more than one site of recurrence.
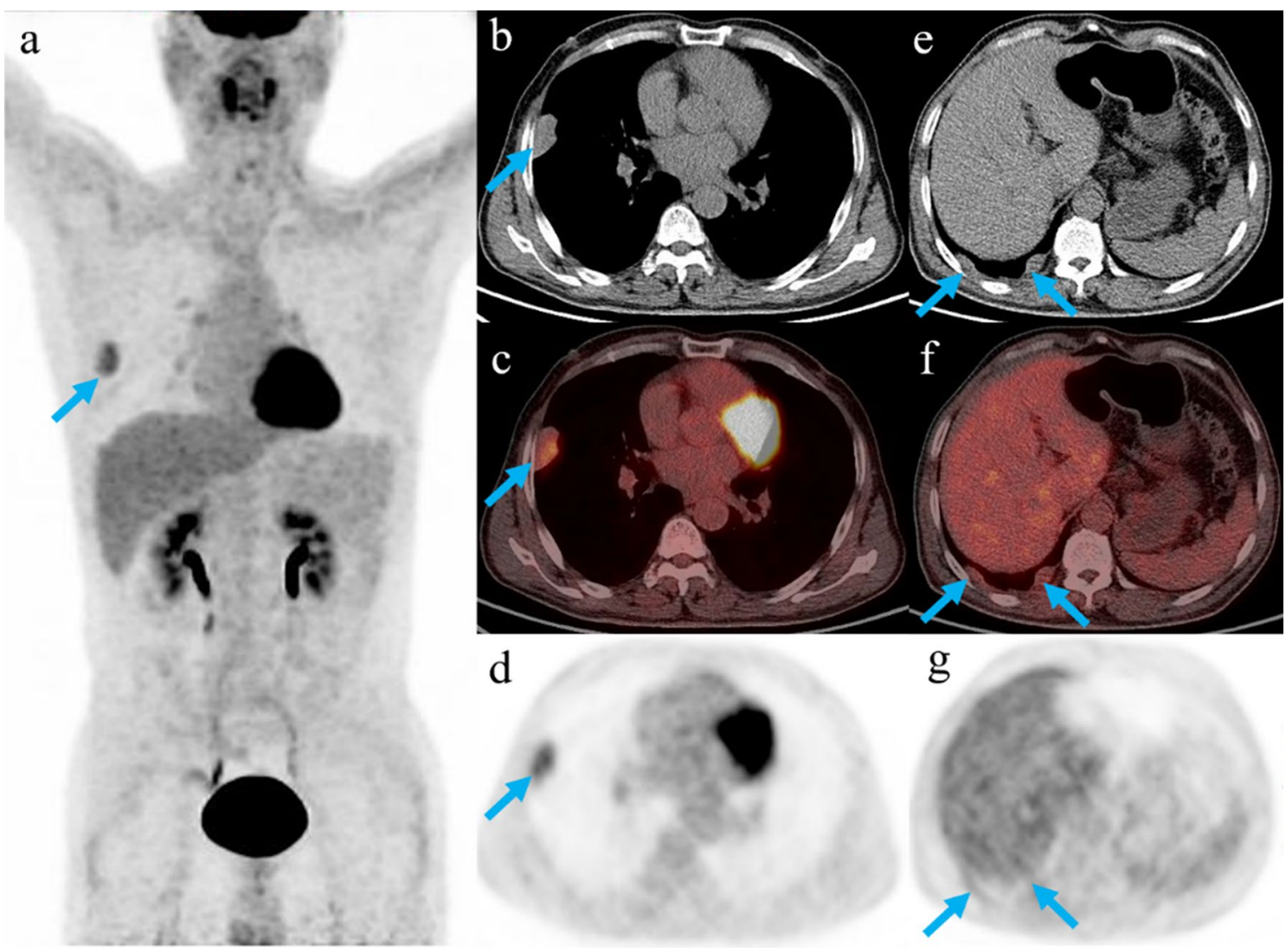

Figure 1. Representative images in a patient who was treated with surgery and radiotherapy for thymoma. Restaging FDG PET/CT showed increased uptake at the right pleura nodule (a-d, arrows; SUVmax, 4.3). In addition, several small-size nodules on the right pleura with negative FDG uptake were also observed on PET/CT (e-g, arrows). The pleural lesions were then surgically resected and confirmed by histopathology as recurrences of thymoma.

PET/CT positive. PET/CT negative patients had significantly longer PFS compared with those who were PET/ CT negative $(\mathrm{P}<0.001)$ (Fig. 2b; Fig. 3). FDG PET/CT also yielded added value if associated with histopathology. Among 50 patients with thymoma, 24 were PET/CT positive, and a negative PET/CT scan was significantly associated with better PFS ( $\mathrm{P}<0.001$; Fig. 2c). Among 33 patients with thymic carcinoma, 26 had positive PET/ CT findings. PET/CT negative patients had significantly better outcomes concerning PFS than PET/CT positive patients $(\mathrm{P}<0.001$; Fig. $2 \mathrm{~d})$.

We also investigated the prognostic value of SUVmax in those 40 patients with true positive results. ROC analysis revealed that the optimal SUVmax cut-off value was $5.3($ AUC $=0.732$; sensitivity $=76.9 \%$; specificity $=0.643$ ) for estimating PFS. Kaplan-Meier survival curve revealed that patients with SUVmax $>5.3$ tended to have shorter PFS than those with SUVmax $\leq 5.3$, while the difference was not statistically significant $(\log -\mathrm{rank}, \mathrm{P}=0.059)$. 


\begin{tabular}{|c|c|c|}
\hline & 2-year PFS (\%) & 3-year PFS (\%) \\
\hline \multicolumn{3}{|l|}{ FDG PET/CT results $(n=83)$} \\
\hline Positive & 57 & 31 \\
\hline Negative & 97 & 91 \\
\hline \multicolumn{3}{|l|}{ Histopathology $(n=83)$} \\
\hline Thymic carcinoma & 61 & 50 \\
\hline Thymoma & 79 & 70 \\
\hline \multicolumn{3}{|l|}{ Masaoka staging $(n=63)$} \\
\hline Stage I/II & 86 & 86 \\
\hline Stage III/IV & 60 & 36 \\
\hline \multicolumn{3}{|l|}{ Histopathology and PET/CT } \\
\hline Positive PET/CT and TC & 26 & 17 \\
\hline Negative PET/CT and TC & 93 & 80 \\
\hline Positive PET/CT and thymoma & 57 & 39 \\
\hline Negative PET/CT and thymoma & 100 & 100 \\
\hline \multicolumn{3}{|l|}{ Masaoka and PET/CT } \\
\hline Positive PET/CT and stage I-II & 66 & 66 \\
\hline Negative PET/CT and stage I-II & 93 & 93 \\
\hline Positive PET/CT and stage III-IV & 33 & 8 \\
\hline Negative PET/CT and stage III-IV & 100 & 83 \\
\hline
\end{tabular}

Table 3. Kaplan-Meir analysis of 2-year and 3-year progression-free survival (PFS).

\section{Discussion}

Limited information is available on the clinical utility of FDG PET/CT for patients with recurrent TETs ${ }^{20}$. The present study demonstrated that FDG PET/CT had a high sensitivity of $100 \%$, but a moderate specificity of $76.7 \%$ for detecting recurrence. Hatem et al. retrospectively evaluated 37 thymoma patients who underwent FDG PET/ CT during postoperative follow-up and revealed a sensitivity of $82 \%$ and specificity of $95 \%{ }^{18}$. Our study demonstrated a higher sensitivity compared to theirs, and this was probably due to the fact that our patient population also included those with thymic carcinoma, which generally had higher FDG activity than thymoma. In their study, the three false-negative results were all due to the small size of pleural nodules, measuring $1.3 \mathrm{~cm}, 2 \mathrm{~cm}$, and $1.2 \mathrm{~cm}$, respectively. As shown in Fig. 1, besides the hypermetabolic pleural lesion, the patient also had several relatively small lesions demonstrating negative uptake of FDG according to visual assessment. The recurrence of TETs on the pleura usually appears as multiple nodules. It might be difficult to determine the actual metabolic rate of those small nodules because of the partial volume effect, which might cause false-negative findings.

As with previous reports, the most common mode of recurrence was pleural recurrence. It is not clear whether pleural recurrences are related to particular biology of the tumor or whether they are the results of the seeding of the tumor ${ }^{21}$. We observed that all 6 patients in our series with distant site recurrences were those with thymic carcinoma. Similarly, Huang et al. also noted that recurrences in thymic carcinoma occurred significantly more frequently at distant sites than in thymoma9. Our analysis showed that the SUVmax of recurrent lesions from thymic carcinoma was significantly higher than that from thymoma. This finding supported the notion that these two thymic tumors are clinically distinct with thymic carcinoma showing more aggressive biomolecular behavior and higher metabolic rate.

FDG PET/CT in the restaging process has recently been demonstrated to play an important role in the prognostic assessment in other types of cancer. While it remains unclear whether restaging FDG PET/CT may also play a role in predicting prognosis in recurrent TETs. Previous studies have indicated the prognostic value of histopathology and Masaoka stage in TETs, ${ }^{6,9,22-25}$. In this study, we evaluated the added prognostic value of FDG PET/CT to histopathology and Masaoka stage. The results demonstrated that in patients with the same Masaoka stage or histopathology, a positive FDG PET/CT scan had a worse outcome concerning PFS than those with a negative scan. Conversely, a negative PET/CT scan allows for the prediction of a more favorable outcome. Negative PET/CT scan might reflect a less aggressive biological nature of TETs, which is associated with better prognosis in terms of PFS. These results suggested that FDG PET/CT can further stratify patients with the same risk of progression and has a higher prognostic value in predicting PFS than Masaoka stage and histopathology alone.

Several limitations of this study should be pointed out. One limitation of the present study was related to the retrospective design. There is a lack of information regarding the presence or the absence of residual tumor after initial surgery, which could have affected the outcome. Second, the variable treatment regimens performed after the restaging scan may also have an impact on the outcome, while an additional analysis grouping patients based on the different treatment strategies was not feasible. Third, Masaoka staging data was not available for all patients as some of the patients received surgery at an outside hospital. The number of patients with stage I/II and positive PET/CT scan was relatively low, which might compromise the power of subgroup analysis. Additional limitation of this study was the small number of enrolled patients. 
a
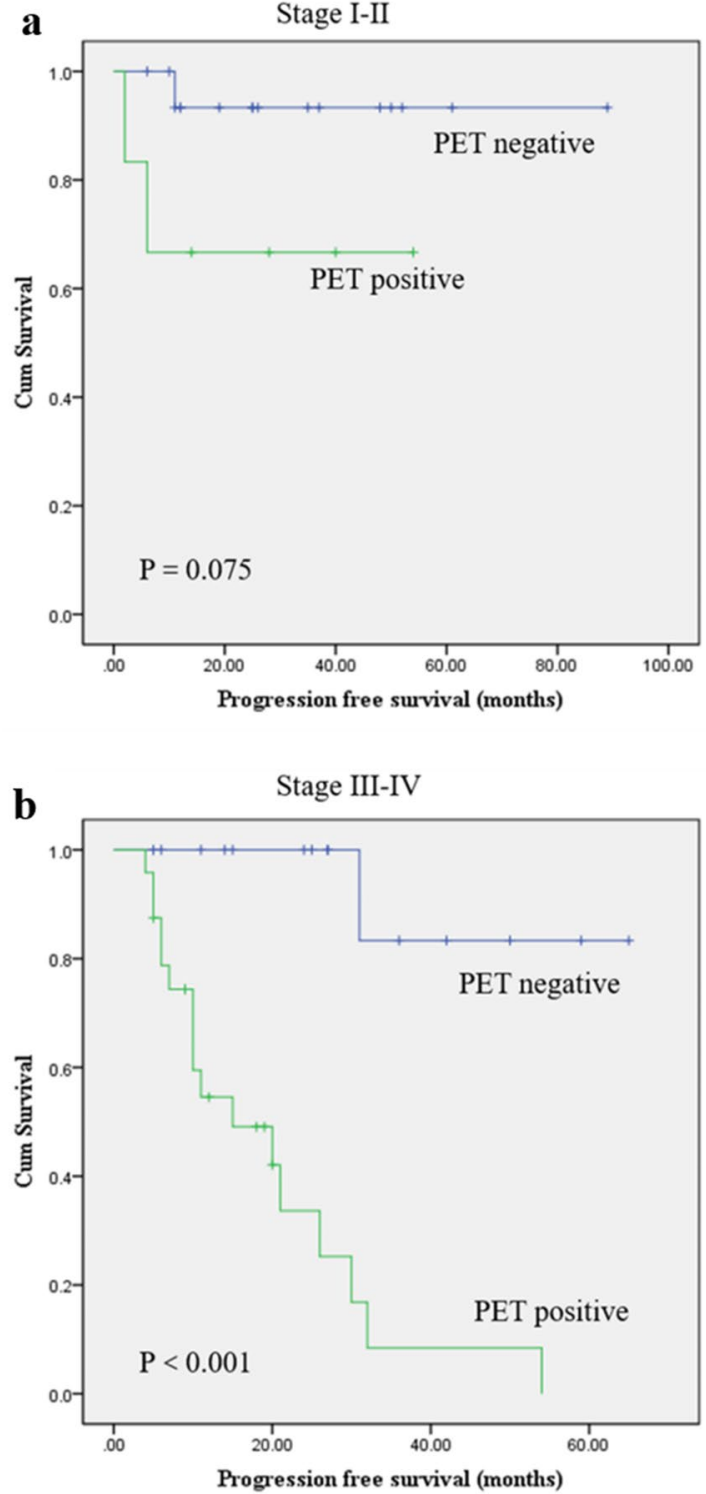

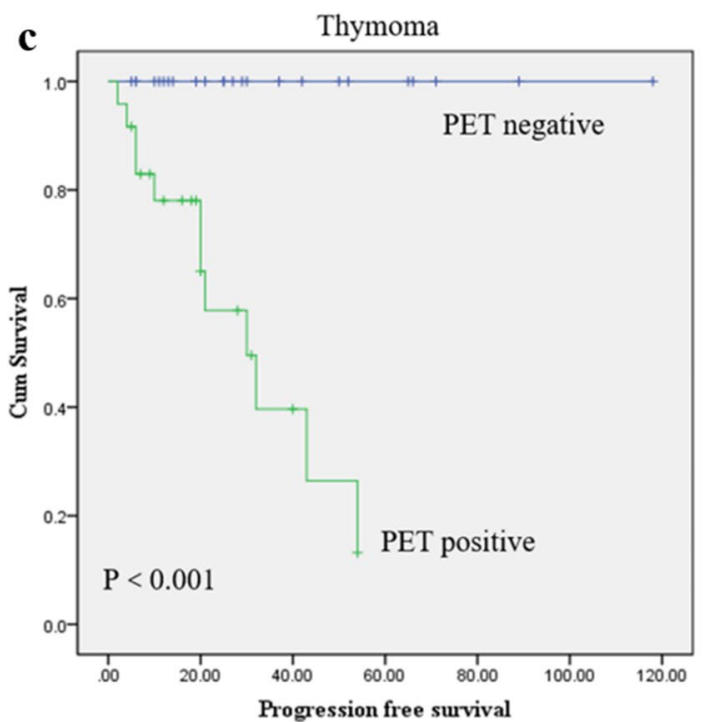

d

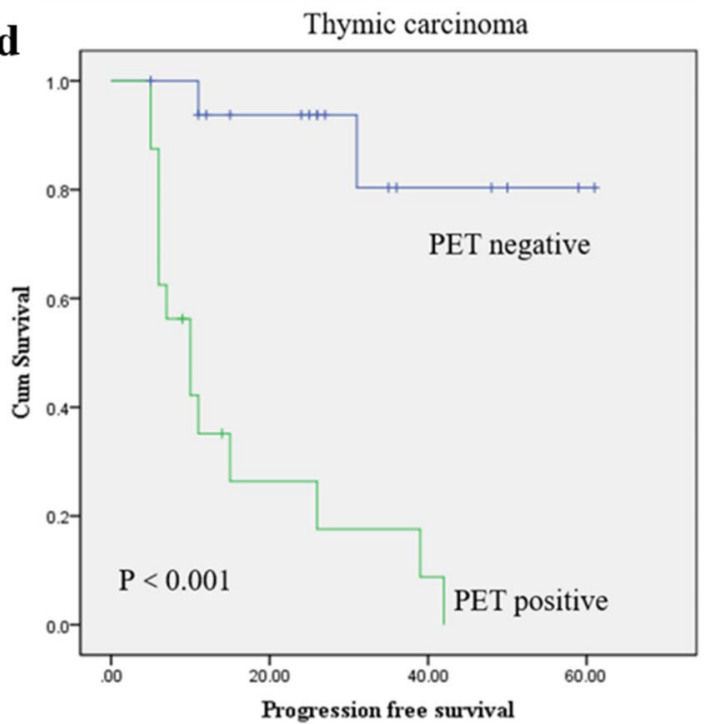

Figure 2. Kaplan Meier curves for progression-free survival (PFS) according to FDG PET/CT positivity for a different stage of Masaoka (a,b) and histopathology $(\mathbf{c}, \mathbf{d})$.

\section{Conclusions}

FDG PET/CT showed a high diagnostic performance in patients with suspicion of recurrent TETs. In addition, FDG PET/CT showed an important prognostic value in assessing PFS.

\section{Methods}

Ethics approval and consent to participate. This study was approved by the Institutional Review Board of Peking Union Medical College Hospital. The requirement for informed consent was waived due to its retrospective nature and it was approved by the IRB of Peking Union Medical College Hospital. Investigations were carried out as per the rules of the Declaration of Helsinki of 1975, revised in 2013. All methods were performed in accordance with the institutional guidelines and regulations.

Patients. Patients affected by TETs after definitive surgery who underwent FDG PET/CT for suspected recurrence between January 2010 and April 2020 were included in this retrospective study. The inclusion criteria were as follows: (1) thymoma or thymic carcinoma was diagnosed according to postoperative pathology; (2) FDG PET/CT was performed for suspicion of recurrence; (3) FDG PET/CT findings were confirmed by histology and/or conventional imaging (CT/MR) performed within 6 months; (4) availability of patients' follow-up for at least 6 months after PET/CT scan; (5) the interval between surgery and FDG PET/CT scan over 4 weeks. A total of 83 patients met these criteria and were included in the final analysis. We performed per-patient analysis for the evaluation of FDG PET/CT performance (e.g., negative and positive scan). For the present study, we only 


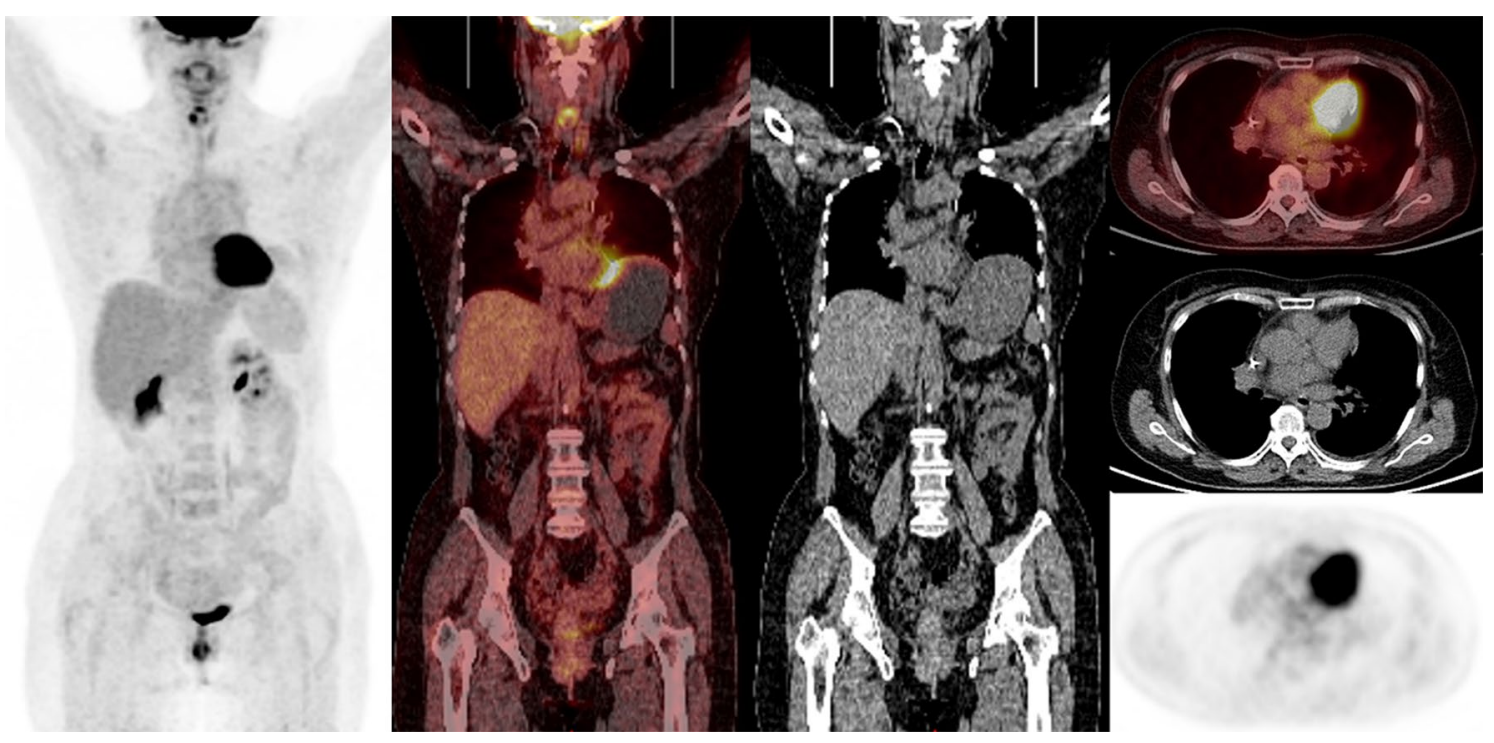

Figure 3. Representative images of an FDG PET/CT scan in a patient who was treated with surgery and adjuvant radiotherapy for stage III thymic carcinoma. Restaging PET/CT was negative. The patient remained disease-free during the follow-up period.

analyzed the first post-treatment FDG PET/CT scan available for each patient; follow-up PET/CT scans were not evaluated for their diagnostic or prognostic value.

Image analysis. All PET/CT images were retrospectively read by 2 experienced nuclear medicine physicians ( $\mathrm{GH}$ and $\mathrm{YJ})$, any disagreements were resolved by consensus. Since there is no reference standard value for maximum standard uptake value (SUVmax), we regard the PET/CT scan as positive based on the qualitative visual assessment. For example, PET/CT scan was defined as positive if the FDG activity in the lesion was moderately or markedly increased (moderate or strong uptake) relative to surrounding soft tissues. Conversely, lesions that showed no or weak FDG uptake (less than or equal to the surrounding soft tissue) were considered negative. SUVmax of the lesion with the highest metabolic activity in the patients with positive PET/CT scan was measured for statistical analysis. The final diagnosis was made by comparing the FDG PET/CT results with histopathology, if available, or with clinical data and/or imaging follow-up for at least 6 months. True positive PET/CT scan corresponded to an abnormal finding confirmed by histopathology or follow-up. PET/CT scan was deemed false positive if the histopathological results from a suspected FDG-positive lesion revealed no evidence of malignancy or the lesion remitted spontaneously without anticancer therapy during the follow-up period. A negative PET/CT scan was considered false negative if the lesion was detected by CT and was confirmed by histopathology or by clinical or imaging progression. True negative PET/CT scan corresponded to the absence of abnormal imaging findings confirmed by negative histopathological results or lack of recurrence during the follow-up. Recurrence patterns were recorded as local recurrence (disease occurring in the anterior mediastinum), regional recurrence (intrathoracic recurrence, including pleural/pericardial dissemination and mediastinal lymph node recurrence), and distant recurrence (intrapulmonary and extrathoracic recurrence) 26,27. $^{26}$.

Statistical analysis. Continuous data are expressed as means and standard deviations. To evaluate the performance of FDG PET/CT in the restaging of TETs, we calculated the sensitivity, specificity, positive predictive value (PPV) and negative predictive value (NPV), and accuracy on a per-patient basis. Mann-Whitney U test was used to compare the variables between different groups. The survival curve of progression-free survival (PFS) was drawn by the Kaplan-Meier method, and differences in survival times between different groups were calculated using log-rank test. PFS was defined as the time from the PET/CT scan to the last follow-up or disease progression. The cutoff value of semi-quantitative parameters was determined by means of receiver-operating characteristic (ROC) analysis. $\mathrm{P}<0.05$ is considered to be statistically significant. The statistical analyses were performed using SPSS (IBM SPSS Statistics for Windows, Version 21.0. Armonk, NY).

\section{Data availability}

The data that support the findings of this study are available from the corresponding author, upon reasonable request.

Received: 17 March 2021; Accepted: 5 October 2021

Published online: 15 October 2021

\section{References}

1. de Jong, W. K. et al. Thymic epithelial tumours: A population-based study of the incidence, diagnostic procedures and therapy. Eur. J. Cancer 44, 123-130. https://doi.org/10.1016/j.ejca.2007.11.004 (2008). 
2. Marx, A. et al. ITMIG consensus statement on the use of the WHO histological classification of thymoma and thymic carcinoma: Refined definitions, histological criteria, and reporting. J. Thorac. Oncol. 9, 596-611. https://doi.org/10.1097/jto.0000000000000154 (2014).

3. Honglin, Y. et al. The correlation of the World Health Organization histologic classification of thymic epithelial tumors and its prognosis: A clinicopathologic study of 108 patients from China. Int. J. Surg. Pathol. 17, 255-261. https://doi.org/10.1177/10668 96909334126 (2009).

4. Conforti, F., Pala, L., Giaccone, G. \& De Pas, T. Thymic epithelial tumors: From biology to treatment. Cancer Treat. Rev. 86, 102014. https://doi.org/10.1016/j.ctrv.2020.102014 (2020).

5. Detterbeck, F. C., Nicholson, A. G., Kondo, K., Van Schil, P. \& Moran, C. The Masaoka-Koga stage classification for thymic malignancies: Clarification and definition of terms. J. Thorac. Oncol. 6, S1710-1716. https://doi.org/10.1097/JTO.0b013e31821e8cff (2011).

6. Mizuno, T. et al. Surgical management of recurrent thymic epithelial tumors: A retrospective analysis based on the Japanese nationwide database. J. Thorac. Oncol. 10, 199-205. https://doi.org/10.1097/jto.0000000000000378 (2015).

7. Kondo, K. \& Monden, Y. Therapy for thymic epithelial tumors: A clinical study of 1,320 patients from Japan. Ann. Thorac. Surg. 76, 878-884. https://doi.org/10.1016/s0003-4975(03)00555-1 (2003) (discussion 884-875).

8. Hamaji, M., Ali, S. O. \& Burt, B. M. A meta-analysis of surgical versus nonsurgical management of recurrent thymoma. Ann. Thorac. Surg. 98, 748-755. https://doi.org/10.1016/j.athoracsur.2014.04.028 (2014).

9. Huang, J. et al. Comparison of patterns of relapse in thymic carcinoma and thymoma. J. Thorac. Cardiovasc. Surg. 138, 26-31. https://doi.org/10.1016/j.jtcvs.2009.03.033 (2009).

10. Venuta, F. et al. Long-term outcome after multimodality treatment for stage III thymic tumors. Ann. Thorac. Surg. 76, $1866-1872$. https://doi.org/10.1016/s0003-4975(03)01020-8 (2003) (discussion 1872).

11. Inoue, A. et al. (18)F-FDG PET for the evaluation of thymic epithelial tumors: Correlation with the World Health Organization classification in addition to dual-time-point imaging. Eur. J. Nucl. Med. Mol. Imaging 36, 1219-1225. https://doi.org/10.1007/ s00259-009-1082-4 (2009).

12. Benveniste, M. F. et al. FDG PET-CT aids in the preoperative assessment of patients with newly diagnosed thymic epithelial malignancies. J. Thorac. Oncol. 8, 502-510. https://doi.org/10.1097/JTO.0b013e3182835549 (2013).

13. Treglia, G. et al. Is (18)F-FDG PET useful in predicting the WHO grade of malignancy in thymic epithelial tumors? A meta-analysis. Lung Cancer 86, 5-13. https://doi.org/10.1016/j.lungcan.2014.08.008 (2014).

14. Ishibashi, M. et al. Usefulness of preoperative (18)F-FDG PET/CT for patients with thymic epithelial tumors. Yonago Acta Med. 62, 146-152. https://doi.org/10.33160/yam.2019.03.020 (2019).

15. Park, S. Y. et al. Value of 18F-FDG PET/CT for predicting the World Health Organization malignant grade of thymic epithelial tumors: Focused in volume-dependent parameters. Clin. Nucl. Med. 41, 15-20. https://doi.org/10.1097/rlu.0000000000001032 (2016).

16. Alongi, P. et al. Recurrent renal cell carcinoma: Clinical and prognostic value of FDG PET/CT. Eur. J. Nucl. Med. Mol. Imaging 43, 464-473. https://doi.org/10.1007/s00259-015-3159-6 (2016).

17. Alongi, P. et al. Recurrent bladder carcinoma: Clinical and prognostic role of 18 F-FDG PET/CT. Eur. J. Nucl. Med. Mol. Imaging 44, 224-233. https://doi.org/10.1007/s00259-016-3500-8 (2017).

18. El-Bawab, H. Y. et al. Clinical use of combined positron emission tomography and computed tomography in thymoma recurrence. Interact. Cardiovasc. Thorac. Surg. 11, 395-399. https://doi.org/10.1510/icvts.2010.236273 (2010).

19. Ito, H. et al. Recurrent thymoma: Radiological (CT and FDG-PET) and histological (WHO criteria) features. Radiat. Med. 24, 292-296. https://doi.org/10.1007/s11604-005-1541-1 (2006).

20. Segreto, S. et al. Evaluation of metabolic response with (18)F-FDG PET-CT in patients with advanced or recurrent thymic epithelial tumors. Cancer Imaging 17, 10. https://doi.org/10.1186/s40644-017-0112-x (2017).

21. Luo, T., Zhao, H. \& Zhou, X. The clinical features, diagnosis and management of recurrent thymoma. J. Cardiothorac. Surg. 11, 140. https://doi.org/10.1186/s13019-016-0533-9 (2016).

22. Wright, C. D. et al. Predictors of recurrence in thymic tumors: Importance of invasion, World Health Organization histology, and size. J. Thorac. Cardiovasc. Surg. 130, 1413-1421. https://doi.org/10.1016/j.jtcvs.2005.07.026 (2005).

23. Blumberg, D. et al. Thymic carcinoma: Current staging does not predict prognosis. J. Thorac. Cardiovasc. Surg. 115, 303-308. https://doi.org/10.1016/s0022-5223(98)70273-9 (1998) (discussion 308-309).

24. Knetki-Wróblewska, M., Kowalski, D. M., Olszyna-Serementa, M., Krzakowski, M. \& Szołkowska, M. Thymic epithelial tumors: Do we know all the prognostic factors?. Thoracic Cancer 12, 339-348. https://doi.org/10.1111/1759-7714.13750 (2021).

25. Tian, W. et al. Surgical outcomes of 215 patients with thymic epithelial tumors: A single-center experience. Thoracic Cancer 11, 1840-1847. https://doi.org/10.1111/1759-7714.13464 (2020).

26. Huang, J., Detterbeck, F. C., Wang, Z. \& Loehrer, P. J. Sr. Standard outcome measures for thymic malignancies. J. Thorac. Oncol. 5 , 2017-2023. https://doi.org/10.1097/JTO.0b013e3181f13682 (2010).

27. Caobelli, F. et al. Predictive value of (18)F-FDG PET/CT in restaging patients affected by ovarian carcinoma: A multicentre study. Eur. J. Nucl. Med. Mol. Imaging 43, 404-413. https://doi.org/10.1007/s00259-015-3184-5 (2016).

\section{Acknowledgements}

This work is supported by National Natural Science Foundation of China (no.81501513), Chinese Academy of Medical Sciences Initiative for Innovative Medicine (CAMS-I2M) 2017-I2M-1-001, and the National Key Research and Development Program of China (No. 2016YFC0901500).

\section{Author contributions}

W.Y.C. and F.L. were responsible for the study design. W.Y.C., F.L., Y.Y.J and G.Z.H. read and approved the final manuscript. Y.Y.J and G.Z.H. contributed equally to this work.

\section{Competing interests}

The authors declare no competing interests.

\section{Additional information}

Correspondence and requests for materials should be addressed to F.L. or W.C.

Reprints and permissions information is available at www.nature.com/reprints.

Publisher's note Springer Nature remains neutral with regard to jurisdictional claims in published maps and institutional affiliations. 
(c) (i) Open Access This article is licensed under a Creative Commons Attribution 4.0 International cc) License, which permits use, sharing, adaptation, distribution and reproduction in any medium or format, as long as you give appropriate credit to the original author(s) and the source, provide a link to the Creative Commons licence, and indicate if changes were made. The images or other third party material in this article are included in the article's Creative Commons licence, unless indicated otherwise in a credit line to the material. If material is not included in the article's Creative Commons licence and your intended use is not permitted by statutory regulation or exceeds the permitted use, you will need to obtain permission directly from the copyright holder. To view a copy of this licence, visit http://creativecommons.org/licenses/by/4.0/.

(C) The Author(s) 2021 